\title{
Coded space-time-frequency OFDM over IEEE 802.11 fading channels
}

\begin{abstract}
In this paper we present 4 transmit and 1 receive (4Tx:1Rx) antenna system of coded spacetime-frequency in conjunction with orthogonal frequency division multiplexing (coded STFOFDM). We concatenate STF block code with channel coding namely convolutional code of rate $1 / 2$ with length 5 to combat channel impairment. We compare our coded STF scheme with ST-OFDM and SF-OFDM over IEEE 802.11 multipath channel under delay spread of $700 \mathrm{~ns}$ and 1 us in outdoor environments. Results show that coded STF-OFDM scheme outperforms other schemes by as much as $8 \mathrm{~dB}$ (against ST-OFDM) in spread of $700 \mathrm{~ns}$. The scheme also outperforms others over a severe channel with delay spread of 1 us but is almost comparable to coded SF-OFDM.
\end{abstract}

Keyword: Coded space-time-frequency; STF-OFDM; IEEE 802.11; ST-OFDM; SF-OFDM 